\title{
Contextual effect of repression of bone morphogenetic protein activity in prostate cancer
}

\author{
Linda Kim Pham 1 , Mengmeng Liang ${ }^{2}$, Helty A Adisetiyo ${ }^{3}$, Chun-Peng Liao ${ }^{2}$, \\ Michael B Cohen ${ }^{4}$, Stanley M Tahara', Baruch Frenkel ${ }^{3}$, Noriyuki Kasahara ${ }^{5}$ \\ and Pradip Roy-Burman ${ }^{2,3}$
}

Departments of ${ }^{1}$ Molecular Microbiology and Immunology ${ }^{2}$ Pathology ${ }^{3}$ Biochemistry and Molecular Biology, Keck School of Medicine, University of Southern California, 2011 Zonal Avenue, Los Angeles, California 90033, USA ${ }^{4}$ Department of Pathology, University of Utah, Salt Lake City, Utah, USA

${ }^{5}$ Department of Medicine, David Geffen School of Medicine, University of California Los Angeles, Los Angeles, California, USA

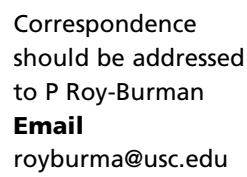

\begin{abstract}
Several studies have focused on the effect of bone morphogenetic protein (BMP) on prostate cancer homing and growth at distant metastatic sites, but very little effect at the primary site. Here, we used two cell lines, one (E8) isolated from a primary tumor and the other (CE1) from a recurrent tumor arising at the primary site, both from the conditional Pten deletion mouse model of prostatic adenocarcinoma. Over-expression of the BMP antagonist noggin inhibited proliferation of $\mathrm{CE} 1$ cells in vitro while enhancing their ability to migrate. On the other hand, $\mathrm{CE} 1 /$ noggin grafts grown in vivo showed a greater mass and a higher proliferation index than the CE1/control grafts. For suppression of BMP activity in the context of cancer-associated fibroblasts (CAFs), we used noggin-transduced CAFs from the same mouse model to determine their effect on E8- or CE1-induced tumor growth. CAF/noggin led to increased tumor mass and greater de-differentiation of the E8 cell when compared with tumors formed in the presence of CAF/control cells. A trend of increase in the size of the tumor was also noted for CE1 cells when inoculated with CAF/noggin. Together, the results may point to a potential inhibitory role of BMP in the growth or re-growth of prostate tumor at the primary site. Additionally, results for CE1/noggin, and CE1 mixed with CAF/noggin, suggested that suppression of BMP activity in the cancer cells may have a stronger growth-enhancing effect on the tumor than its suppression in the fibroblastic compartment of the tumor microenvironment.
\end{abstract}
Key Words
- BMP
- noggin
- prostate cancer mouse model
- prostate cancer cell lines
- cancer-associated fibroblasts

Endocrine-Related Cancer (2013) 20, 861-874

\section{Introduction}

Previously, our laboratory showed that bone morphogenetic proteins (BMPs), such as BMP2 and BMP7, could enhance certain in vitro oncological characteristics of human prostate tumor cell lines (Yang et al. 2005, 2006, 2008, Lim et al. 2009). However, there is still a significant degree of inconsistencies reflected in the literature.
For example, while there are reports (Feeley et al. 2006, Kwon et al. 2010) that concurred with our observations that BMP treatment promotes migration and invasion of PC-3 human prostate cancer cells in vitro, there are also reports implying that BMP treatment inhibits migration and invasion of these same cells (Buijs et al. 2007,

Published by Bioscientifica Ltd 
Ye et al. 2007, Benelli et al. 2010). It appears that a study of a single BMP may not be the most effective approach as individual BMP family members may differ in their ability to confer positive or negative effects. To assess the overall effect of BMP signaling in prostate cancer, we used noggin, an inhibitor of multiple BMPs, to investigate how loss of BMP signaling might influence in vitro and in vivo growth characteristics of prostate cancer cell lines.

Noggin is a secreted glycoprotein with a monomer molecular weight of $32 \mathrm{kDa}$, but usually exists as a homodimer (Yanagita 2005, Krause et al. 2011). Like other BMP antagonists, it has a cysteine-rich C-terminal region that, via cysteine knots, confers a distinct ring structure used to classify the antagonists into three subfamilies. noggin, with its ten-member ring structure, belongs to the chordin and noggin family (AvsianKretchmer \& Hsueh 2004). Crystal structures of both BMP7 and noggin showed that noggin binding to BMP7 occluded both type I and II BMP receptor binding sites on BMP7. This prevented BMP7, and presumably other BMPs to which noggin binds, from activating both SMADdependent and SMAD-independent signaling pathways via the BMP receptors (Groppe et al. 2002).

Noggin was reported to bind with varying affinities to BMPs 2, 4, 5, 6, 7, 13, and 14 (Krause et al. 2011). Song et al. (2010) demonstrated that noggin binds to BMP2 and BMP4 more strongly than BMP7 and to BMP6 with only low affinity. Shaw et al. (2009) reported that under their culture conditions, noggin impeded BMP4-dependent but not BMP7-dependent gene transcription in LNCaP cells. Notably, another BMP antagonist, sclerostin (SOST), was shown to bind noggin, although in this case the SOSTnoggin complex was mutually inhibitory and increased BMP availability (Winkler et al. 2004).

Yuen et al. (2008) showed that while BMP6 expression by itself was not a good prognostic indicator of distant metastasis of prostate tumors, combining high BMP6 expression with low noggin and SOST expression was a more reliable predictor. In vitro data showed that adding noggin to conditioned media from prostate cancer cell lines reduced their ability to induce osteoblastic activity (Dai et al. 2004, 2005). Consistent with these observations, Schwaninger et al. (2007) showed that osteolytic cell lines (PC-3, PC-3M-Pro4) expressed noggin while osteoblastic cell lines (LNCaP, C4-2, C4-2b) did not. These in vivo data were mostly derived from implantation of noggin over-expressing cell lines into bone. Regardless of the osteoblastic or osteolytic nature of the prostate tumor cell line, osteoblastic/-lytic response was reduced, although tumor growth was not always necessarily inhibited
(Feeley et al. 2005, 2006, Schwaninger et al. 2007, Virk et al. 2009, 2010). Notably, a recent paper showed that noggin silencing in PC-3 cells preserved bone formation in the osteolytic lesions and decreased tumor growth (Secondini et al. 2011). To date, most noggin studies used prostate cancer cell lines isolated from metastatic lesions and are limited to its influence in the bone microenvironment. In this study, we used a newly established murine prostate adenocarcinoma cell line, isolated from a prostate tumor in the androgen-dependent phase, as well as a cell line from a recurrent tumor at the primary site after castration to study how the overall inhibition of BMP signaling in either the neoplastic epithelium or the fibroblastic stromal compartment of the prostate cancer might affect tumor growth.

\section{Materials and methods}

\section{Cell lines}

E8 cell line was derived from an intact Pten deletion mouse tumor following a similar procedure as described for generation of E2 and $\mathrm{E} 4$ cell lines (Liao et al. 2010). Both E8 and another line, cE1 (Liao et al. 2010) derived from a castration-resistant prostate cancer from the same model, were maintained at $5 \% \mathrm{CO}_{2}, 37^{\circ} \mathrm{C}$, in DMEM supplemented with $10 \% \mathrm{FBS}, 25 \mu \mathrm{g} / \mathrm{ml}$ bovine pituitary extract (Invitrogen), $5 \mu \mathrm{g} / \mathrm{ml}$ insulin (Sigma-Aldrich), and $6 \mathrm{ng} / \mathrm{ml}$ recombinant human epidermal growth factor (Invitrogen). cE1 cells were later switched to a medium similar to the above with the addition of $1 \mathrm{nM}$ R1881 (Perkin Elmer, Waltham, MA, USA) and the replacement of 10\% FBS with $10 \%$ charcoal:dextran-stripped serum (Gemini, Sacramento, CA, USA) to reduce endogenous androgens. Cancer-associated fibroblast (CAF) cells were isolated and cultured as described previously (Yang et al. 2008).

\section{Lentivirus production}

The plasmid containing Noggin cDNA was a kind gift from Dr Cheng-Ming Chuong of the University of Southern California. The lentivirus construct (Fig. 1A) was prepared at the UCLA Vector Core. The open reading frame (ORF) of $m$ Noggin was amplified with primers $5^{\prime}$-mNoggin-BamHI (GATCGGATCCATGGAGCGCTGCC) and $3^{\prime}$-mNogginEcoRI (GCTAGAATTCCTAGCAGGAACACTTACACTCG) using Phusion HF PCR mix (Thermo Scientific, Pittsburgh, PA, USA). PCR product was gel isolated and digested with BamHI-HF and EcoRI-HF (New England Bioscience, Ipswich, MA, USA). pRRL-sin-cPPT-MCS-IRES-emdRFP

Published by Bioscientifica Ltd. 


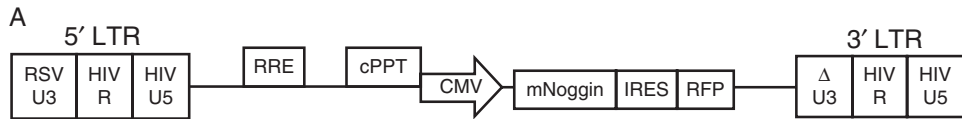

B
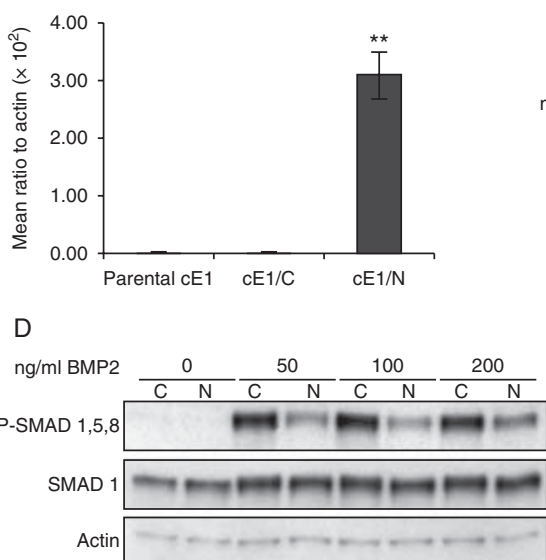

$\mathrm{F}$

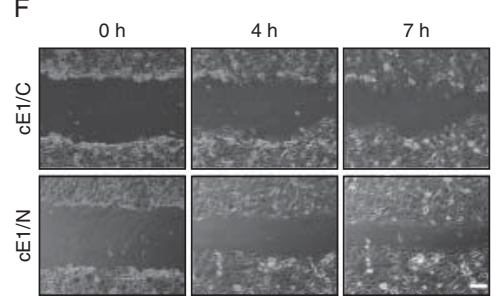

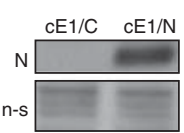

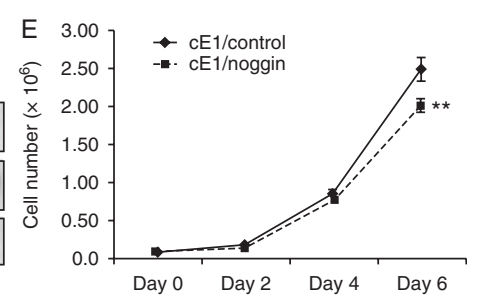

G

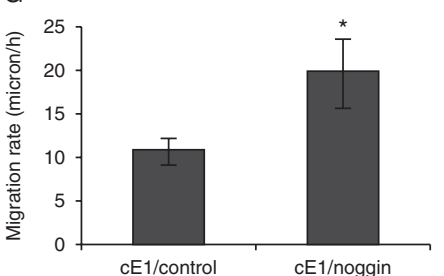

\section{Figure 1}

Over-expression of noggin in CE1 cells suppresses proliferation but increases migratory ability. (A) Schematic diagram of the vector carrying murine Noggin. pRRL-sin-CPPT-MCS-IRES-emdRFP plasmid is a thirdgeneration lentiviral vector containing a chimeric $5^{\prime}$ LTR (made of Rous sarcoma virus U3 and HIV-1 R/U5 regions), a central polypurine tract (cPPT)/central termination sequence, and an immediate early cytomegalovirus (CMV) promoter. RRE, rev response element. (B) qPCR data showing that Noggin mRNA is significantly upregulated in noggin-RFP-transduced cells compared with the parental and RFP control cells. (C) Western blot of the conditioned medium collected from CE1/control (CE1/C) and CE1/noggin (CE1/N) cell lines showing increased noggin secretion in CE1/noggin vs cE1/control. Medium was collected from cells cultured in DMEM for $24 \mathrm{~h}$.

plasmid, a third-generation lentiviral construct kindly given by Dr Luigi Naldini of the University of Milan, Italy (Zufferey et al. 1998), was cut at the multiple cloning site with the same enzymes. Two products were ligated and transformed in STBL3 cells. Lentivirus was produced in $293 \mathrm{~T}$ cells using a third-generation packaging system as described previously (Dull et al. 1998).

\section{Lentivirus infection}

E8 and cE1 cells were plated at 100000 cells/well in sixwell plates and incubated with $1 \mathrm{ml}$ growth medium containing lentiviruses, at an MOI (multiplicity of
$\mathrm{N}$, noggin; n-s, non-specific bands. (D) Western blot showing that overexpressed noggin suppressed SMAD 1,5,8 phosphorylation induced by 0 50,100 , and $200 \mathrm{ng} / \mathrm{ml}$ BMP2. cE1 cells were cultured in $0.1 \%$ serum overnight and then treated with BMP2 for $1 \mathrm{~h}$ before cell lysate was collected. (E) CE1/control and CE1/noggin cell growth in maintenance media was determined by counting cells at the indicated intervals. (F) Representative $100 \times$ brightfield micrographs to indicate increased motility of CE1 cells over-expressing noggin. Bar, $300 \mu \mathrm{m}$. (G) Quantitation of the migration rate in the wound-healing assay. Using pictures taken at zero and $4 \mathrm{~h}$, the gap distance was measured in microns and divided by time in hours. Shown are mean \pm s.D. ${ }^{\star} P<0.05$. ${ }^{*} P<0.01$.

infection) of 100 , in the presence of $5 \mu \mathrm{g} / \mathrm{ml}$ polybrene overnight. The next morning, wells were washed with PBS twice and fed with complete medium. The procedure was repeated once more and then the transduced cells were sorted by flow cytometry on the basis of red fluorescent protein (RFP) fluorescence.

\section{RNA preparation and real-time RT-PCR}

Total RNA was extracted using the Tissue RNA miniprep kit with DNase I set (Bioland, Paramount, CA, USA) following the recommended protocol by the manufacturers. Isolated RNA (1 $\mu \mathrm{g})$ was reverse transcribed using

Published by Bioscientifica Ltd. 
qScript cDNA Synthesis Kit (Quanta, Gaithersburg, MD, USA). Real-time PCR was carried out using $12.5 \mu 1$ FastStart Universal SYBR Green Master (Roche) with $1 \mu$ l cDNA in a total volume of $25 \mu$ l. The PCR conditions were as follows: one cycle of $95^{\circ} \mathrm{C}$ for $10 \mathrm{~min} ; 40$ cycles of $95^{\circ} \mathrm{C}$ for $30 \mathrm{~s}$, $55^{\circ} \mathrm{C}$ for $1 \mathrm{~min}$, and $72{ }^{\circ} \mathrm{C}$ for $30 \mathrm{~s}$; and one cycle of $95^{\circ} \mathrm{C}$ for $1 \mathrm{~min}$ and $55^{\circ} \mathrm{C}$ for $30 \mathrm{~s}$. Reactions were carried out with the Stratagene Mx3000P PCR machine, and the cycle thresholds were determined with the accompanying software. Actin was used for each sample as internal control. Real-time PCR primers are listed in Table 1.

\section{Analysis of proliferation}

Growth curves in serum-free media with varying amounts of R1881 were obtained as described previously (Liao et al. 2010). Growth of transduced cell lines was determined by plating 50000 cells/well in growth medium into six-well plates (day 0). Medium was refreshed every 2 days and the proliferation rate was determined by cell counting at the indicated time points using a Beckman Coulter Counter.

\section{Immunostaining}

Immunostaining was done according to previously published methods (Liao et al. 2010). For immunohistochemistry, the following primary antibodies were used: androgen receptor (AR) (1:200; Santa Cruz, Dallas, TX, USA),
CK8 (TROMA-1) (1:100; Developmental Studies Hybridoma Bank, University of Iowa, IA, USA), CK5 (1:1000; Covance, Princeton, NJ, USA), vimentin (1:50; Cell Signaling, Beverly, MA, USA), Ki67 (1:200; Vector Laboratories, Burlingame, CA, USA), or CD31 (PECAM-1) (1:1000; Santa Cruz). Secondary antibody consisted of biotinylated goat, rabbit, or rat IgG (1:200; Vector Laboratories). For vimentin and CK8 co-immunofluorescence staining, sections were incubated in the above vimentin and CK8 antibody overnight and then stained with FITCconjugated anti-rat IgG (1:80; Sigma-Aldrich) as well as TRITC-conjugated anti-rabbit IgG (1:50; Sigma-Aldrich). Negative controls were done under the same protocol with the omission of the fluorescing antibody. Ki67 quantitation was performed by counting positively stained cells in three $400 \times$ fields per graft and divided by the number of nuclei found in those fields. CD31 quantitation involved counting the number of positive structures per $400 \times$ field, in triplicate. Calculation of areas containing glandular structure involved taking low-magnification images of the entire graft and using Image to quantitate areas with glandular structure.

\section{Western blot}

Cell lysates and conditioned medium were collected as described previously (Yang et al. 2005). Primary antibodies were used as follows: goat anti-noggin (1:1000; R\&D

Table 1 List of primers used for RT-PCR of various mouse BMPs and their receptors

\begin{tabular}{llll}
\hline Gene & Forward primer & Reverse primer \\
\cline { 2 - 3 } Noggin & TGTACGCGTGGAATGACCTA & TGAGGTGCACAGACTTGGA \\
Sost & ATGACGCCAAAGATGTGTCCGAT & CACCACTTCACGCGCCCGAT \\
Bmp 2 & AGATCTGTACCGCAGGCACT & CCGTTTCCCACTCATCTCT \\
Bmp 4 & TGAGCCTTCCAGCAAGTTT & CTTCCCGGTCTCAGGTATCA \\
Bmp6 & CATGGTCATGAGCTTGTCA & CAAGTCTTGCAGGAGCATCA \\
Bmp7 & CAAGTCTTGCAGGAGCATCA & CAAGTCTTGCAGGAGCATCA \\
Bmp9 & GAAAACAGCAGCAGTGACCA & GGTGGCGTTCATGTAGGAGT \\
Bmp10 & AGGAGACCTGGAAGGGTTA & AGTTTCTGCCTGGTTTCCTG \\
Bmp11 & ATTCGCCACAGACCGGACCTCC & CAACCGCAGTTCAGCCATGACG \\
Bmp12 & ACCACCGAGACGGTCATAAG & GGCCTTCAGTACCTTGGTGA \\
Bmp13 & GATGTCGCTTTACAGGAGCC & ACGTCGAACAGGAAGCTCTG \\
Bmp14 & CGCGTGGTGCCTCACGAGTA & GGAGTGTGCGAGAGATCGTCCAGT \\
Alk1 & TCGAGAGCCCAAGGAGCCGTT & GCAGGGCCTCGGTCATCTTGCC \\
Alk2 & CGGCTCTGGACGTGAGAC & GGTGAGATCTGCAAAACGTG \\
Acvr2a & TGCTAATGATGATGGCTTTCC & CCTTCACAGTGGTCCTCGTT \\
Acvr2b & AGCGAGAACTTCCTACGGCT & CCTGAGTTTCTGATCTGCCA \\
Bmpr1a & CGACAAGGGCTCCCTCACGGA & GCCCTCACCACGACACCACG \\
Bmpr1b & ATGCAAGGATTCACCGAAAG & AACAACAGGGGGCAGTGTAG \\
Bmpr2 & CTCCCTCTGCTGGTCCAAAGGACA & CCAGCTGGCTTCCTCCGTGGT \\
\hline
\end{tabular}

All primer sets were designed by the authors using Primer3 Software with the exception of the Noggin primer set, which was taken from Reinhold et al. (2004).

http://erc.endocrinology-journals.org DOI: 10.1530/ERC-13-0100
(C) 2013 Society for Endocrinology Printed in Great Britain
Published by Bioscientifica Ltd. 
Systems, Minneapolis, MN, USA), rabbit anti-SMAD1 (1:1000; Cell Signaling Technology), anti-phosphoSMAD 1,5,8 (1:1000; Cell Signaling Technology), and goat anti-actin (1:5000; Santa Cruz).

\section{Migration assay}

E8, cE1, and CAF cells were allowed to reach $90-100 \%$ confluency in six-well plates. The growth medium was saved and replaced with fresh medium containing $10 \mu \mathrm{g} / \mathrm{ml}$ of mitomycin C (Sigma-Aldrich). The cells were incubated at $37^{\circ} \mathrm{C}$ for $2 \mathrm{~h}$, after which the wound line was made with a $200 \mu$ pipette tip on the monolayer cultures of the cells. Cells were washed with PBS twice, the saved growth media was added, and photographs were taken at indicated time points. Gap distance was measured using Image Software and divided by elapsed time to obtain migration rate.

\section{Tumorigenicity assay}

Transduced E8 or cE1 $\left(1 \times 10^{6}\right)$ cells were suspended in $50 \mu \mathrm{l}$ growth media and mixed with $50 \mu \mathrm{l}$ Matrigel (BD Bioscience, San Jose, CA, USA) and then inoculated subcutaneously into NOD.SCID mice of 8-12 weeks of age. Grafts were collected surgically at 6 weeks postinoculation from the killed animals. For assays involving $\mathrm{CAF}, 2 \times 10^{6}$ transduced CAF cells were added to $1 \times 10^{6} \mathrm{E} 8$ or cE1 in $100 \mu$ l growth media and were treated as mentioned earlier.

\section{Statistical analysis}

Results were evaluated as the mean \pm s.D. of at least two different experiments performed in triplicate. Differences between individual groups were analyzed by Student's $t$-test. $P$ values of $<0.05$ were considered statistically significant.

\section{Results}

\section{Signaling, growth, and migration of noggin-transduced CE1 cell line}

Stable cell lines of noggin over-expressing cE1 cells were created via lentivirus-mediated infection of either nogginRFP or RFP alone (Fig. 1A). The former is referred to as cE1/noggin and the latter cE1/control. After FACS sorting, over-expression of noggin was confirmed by both qPCR and western blot. qPCR analysis showed that Noggin transcript in cE1/noggin cells was $>10000$-fold higher than in parental cE1 and cE1/control cells (Fig. 1B) and the noggin western blot confirmed that a similar pattern of expression could also be detected in the conditioned medium (Fig. 1C). It should be noted that conditioned medium from control cells did not show any detectable amount of noggin. After confirming over-expression of noggin, we tested the ability of noggin to antagonize BMP2 signaling. Immunoblotting for phospho-SMAD 1,5,8 demonstrated that cE1/control had little to no activation of the SMAD pathway. The addition of BMP2induced strong phosphorylation of SMAD 1,5,8 in cE1/control cells, and, as expected, expression of noggin reduced the phosphorylation of SMAD and increasing concentrations of BMP2 were able to partially alleviate the repression of SMAD activation (Fig. 1D).

As it is known that forced expression of BMPs induces noggin expression and that knockdown of BMPs decreases noggin (Haudenschild et al. 2004, Ye et al. 2007), we checked whether noggin over-expression might affect BMP expression in cE1 cells. We performed qPCR analysis and saw that the BMP profile for CE1/control was similar to that of cE1/noggin. Of the ten BMPs assayed, only Bmp4 showed significantly higher transcript levels in cE1/noggin vs cE1/control. Sost expression was also assessed, as we wanted to know the likelihood of noggin function being antagonized and we saw that transcript levels were similarly low in both cell lines (data not shown). We also assayed the expression of the four type I (Bmpr1a, Bmpr1b, Alk1, and Alk2) and three type II (Bmpr2, Acvr2a, and Acvr2b) BMP receptors known to bind BMPs and found that both cell lines expressed Bmpr1a and Bmpr2 the highest. There was also modest expression of Alk2, Acvr2a, and Acvr2b. There was little to no expression of Bmpr1b and Alk1. Overall, the over-expression of noggin did effect expression of BMPs and BMPRs in cE1 cells (data not shown).

Next, we assayed whether noggin over-expression influenced any characteristics known to contribute to oncological properties. We performed proliferation and migration assays with cE1/control and cE1/noggin lines. Figure $1 \mathrm{E}$ shows that noggin over-expression slowed cell growth and by day 6 , the number of cells in the cE1/noggin plates was significantly less $(P<0.01)$ than in cE1/control. Cell cycle analysis shows that forced noggin expression caused a small but consistent increase in G0/G1 phase cE1 cells, which is consistent with the change in growth rate, although this was not statistically significant (data not shown). To assess migration, a wound-healing assay was done and as Fig. 1F illustrates, it was found that cE1/noggin cells penetrated the wound line and closed the

Published by Bioscientifica Ltd. 
gap faster than cE1/control cells. Using ImageJ, we measured the gap over time and found that the migration rate for cE1/noggin cells was significantly faster than cE1/control cells (Fig. 1G).

\section{Forced expression of noggin increases tumor proliferation in vivo}

In vitro data supported a role of BMP signaling in promoting proliferation but not migration of cE1 cells. To determine whether these observations would be supported in vivo, we subcutaneously implanted $1 \times 10^{6}$ cE1/control or cE1/noggin cells into NOD.SCID mice and harvested the resultant grafts after 6 weeks. Tumor incidence was 100\% $(6 / 6)$ for both cell lines. However, in contrast to the in vitro growth rates, the tumors composed of cE1/noggin cells were almost twofold larger than those of cE1/control cells (Fig. 2A). Histopathological analysis revealed that both cE1/control grafts and cE1/noggin grafts were of similar phenotype. In both types of grafts, keratin 8 (CK8), AR double-positive cells formed glandular-like structures (Fig. 2B). AR staining was confined largely to the nucleus of CK8-positive cells as was expected. Vimentin staining was present in the stroma, staining both fibroblasts and capillary structures and, for the most part, not detected in epithelial structures. Staining for Ki67, a cell proliferation marker, was abundant in all grafts with at least $10 \%$ of all cells staining positive. Consistent with Fig. 2A, the Ki67 proliferation index was significantly higher in cE1/noggin vs cE1/control cells (Fig. 2C).

Previously, we described that BMPs not only affected carcinoma cells but also contributed to tumor progression
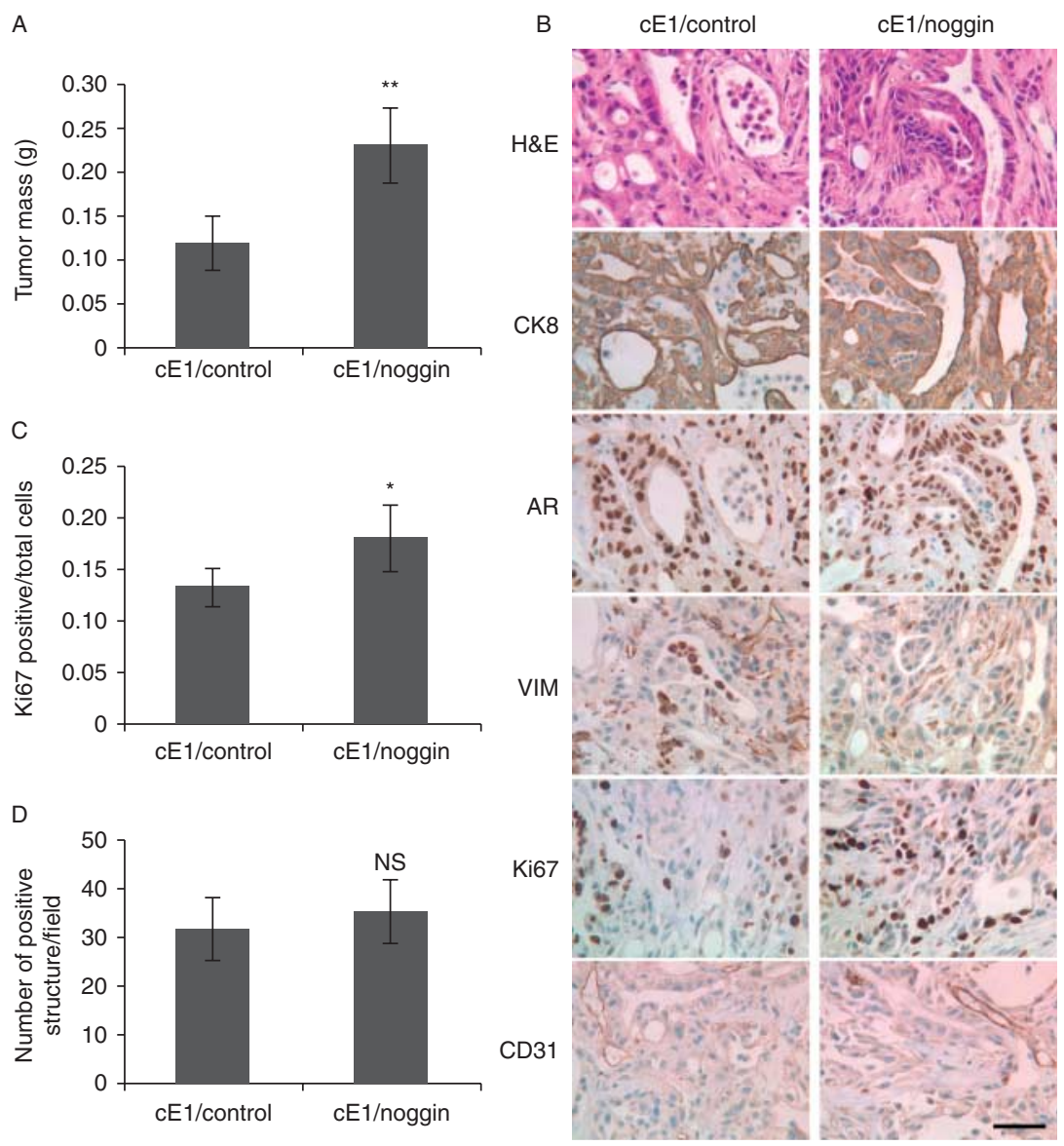

\section{Figure 2}

Histological analyses of tumors induced by the $\mathrm{CE} 1 /$ control or $\mathrm{CE} 1 /$ noggin cell lines in male NOD.SCID mice. (A) Bar graph showing significantly higher tumor mass (g) was seen for CE1/noggin grafts vs CE1/control. Shown values are mean \pm S.D. (B) $400 \times$ micrographs showing $H \& E$ and IHC staining of sections from $C E 1 /$ control and $C E 1 /$ noggin grafts for $C K 8, A R$, vimentin, Ki67, and CD31. Bar, $50 \mu \mathrm{m}$. (C) Bar graph of Ki67 quantitation. Positive

http://erc.endocrinology-journals.org DOI: $10.1530 /$ ERC-13-0100
(C) 2013 Society for Endocrinology Printed in Great Britain nuclei were counted in three random $400 \times$ fields and averaged. Shown are mean values \pm s.D. (D) Bar graph showing quantitation of CD31 IHC staining. Shown are mean values \pm s.D. The number of positive structures in three independent $400 \times$ fields in each graft was counted and averaged. ${ }^{\star} P<0.05$. ${ }^{*} P P<0.01$. NS, not significant. 
by signaling to the stroma. We demonstrated that BMP treatment of CAFs induced SDF1 expression which, in turn, triggered increased tube formation in an in vitro matrigel angiogenesis assay (Yang et al. 2008). In order to evaluate whether disruption of BMP signaling via noggin secretion from the epithelial component could affect angiogenesis in vivo, we did an immunohistochemical analysis of CD31 expression. As Fig. 2B and D illustrate, there was no significant difference in the number of positive CD31 structures among cE1/control or cE1/ noggin grafts, indicating that angiogenesis was not significantly impacted.

\section{Expression of noggin in CAF cells}

We published evidence that the effects of BMP were seen not only on cancer cells but also on tumor-associated fibroblastic cells (Yang et al. 2008). Our in vivo evidence indicated that while transduction of noggin into carcinoma cells did affect tumor growth, the histopathology of the tumor remained unaffected. To determine the effect of noggin over-expression in the stromal compartment, we first transduced primary CAF cultures with RFP or nogginRFP. The primary CAFs were derived from the prostate of the same Pten knockout mouse model as the tumor cell line. Unlike the malignant epithelial cells, they did not harbor the Pten deletion. We believe that using these cancer cells and fibroblasts would be representative of the tumor microenvironment of the same mouse model. However, these CAFs and tumor cell lines were derived from different tumors and as such have a similar but not identical genetic background. Figure $3 \mathrm{~A}$ shows that conditioned medium collected from $\mathrm{CAF} /$ noggin cells contained significant amounts of noggin protein while conditioned media from $\mathrm{CAF} /$ control cells showed no detectable expression of noggin. BMP2 treatment of $\mathrm{CAF} /$ control cells showed a significant increase in phospho-SMAD 1,5,8 compared with CAF/noggin, although some activation of SMAD was present in all noggin lanes (Fig. 3B). Increasing concentrations of BMP2 were able to partially counteract the effect of noggin. In order to ascertain the effect of BMP on CAF, we again examined cell proliferation and migration in control and noggin lines. Over-expression of noggin in CAF did decrease CAF cell proliferation as seen in cE1 cells (Fig. 3C). Unlike cE1 cells, CAF migration was not affected by the presence of noggin (Fig. 3D and E).

\section{Characterization of a new murine prostate epithelial cell line}

A new line of androgen-dependent prostate cancer cells was established from the conditional Pten-null mouse model. This cell line, dubbed E8, was isolated in the same

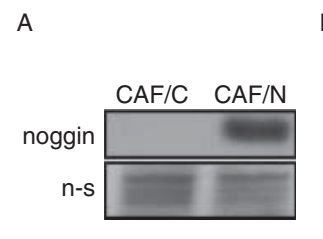

D

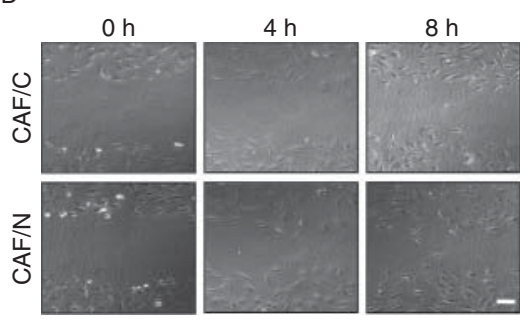

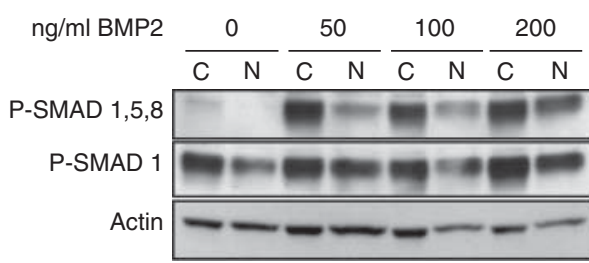

E
C

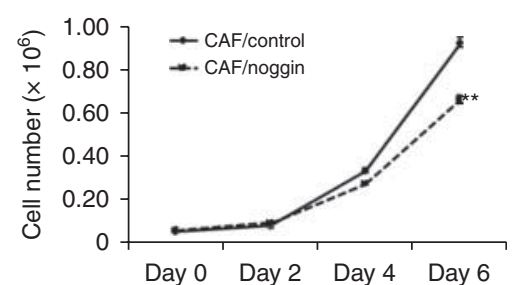

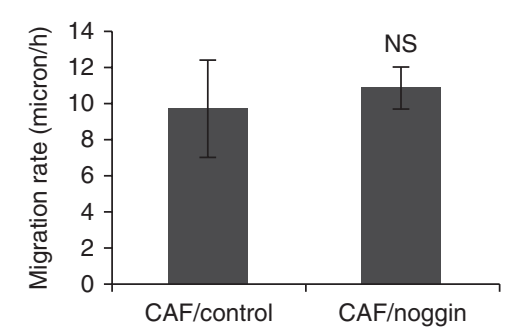

\section{Figure 3}

Noggin over-expression in CAF cultures reduces growth but not migration in vitro. (A) Western blot of conditioned media collected from CAF/control and CAF/noggin cell lines showing increased noggin secretion in CE1/noggin vs CE1/control lanes. N, noggin; n-s, non-specific bands.

(B) Western blot showing that over-expressed noggin suppresses SMAD 1, 5,8 phosphorylation. CAF cells were cultured in $0.1 \%$ serum overnight and then treated with $0,50,100$, or $200 \mathrm{ng} / \mathrm{ml} \mathrm{BMP} 2$ for $1 \mathrm{~h}$ before the cell

http://erc.endocrinology-journals.org DOI: $10.1530 /$ ERC-13-0100
(C) 2013 Society for Endocrinology Printed in Great Britain lysate was collected. (C) Growth curve of CAF/control and CAF/noggin cell lines as determined by counting the cell numbers every 2 days. (D) Representative $100 \times$ brightfield micrographs of migration assay showing no difference in rate. Bar, $300 \mu \mathrm{m}$. (E) Quantitation of the migration rate in the wound-healing assay. Using pictures taken at 0 and $4 \mathrm{~h}$, the gap distance was measured in microns and divided by time in hours. Shown are mean values \pm s.D. ${ }^{*} P<0.01$. NS, not significant. 
manner as previously published cell lines, E2 and E4 (Liao et al. 2010). E8 cells appeared polygonal with somewhat spindle-shaped morphology and grew very rapidly; unlike cE1 cells, they did not grow in clumps (Fig. 4A). Using realtime PCR, we checked for the expression of epithelial markers, Ck8, p63, and E-cadherin (Fig. 4B). We saw that there was high expression of $C k 8$ and no detectable expression of $p 63$, likening the cells to luminal epithelial cells. E-cadherin expression was only moderate, consistent with the lack of cell-to-cell adhesion. As other androgendependent cell lines isolated from this mouse model have a tendency to undergo an epithelial-mesenchymal-like transition (EMT), we also checked E8 cells for expression of fibroblast and EMT markers. We found that there was high expression of vimentin and moderate expression of $\mathrm{N}$-cadherin, indicating that some de-differentiation occurred, but little to no expression of EMT transcription factors, such as, Twist, Snail, and Snai2 (Slug) (Fig. 4C). It is also interesting to note that E-cadherin was expressed at a similar level to $\mathrm{N}$-cadherin, further demonstrating the de-differentiated nature of these cells. E8 cells were isolated from an intact mouse and should be androgen dependent. Western blot analysis show that E8 cells express androgen receptor levels similar to E2 and E4, cell lines also isolated from an androgen-dependent tumor from the same mouse model. To determine how proliferation would be affected by the presence of androgens, growth assays were done in a modified serum-free media containing 0 , 1, or $5 \mathrm{nM}$ R1881. Figure 4D shows that in the absence of androgen, E8 proliferation was inhibited. In fact, practically, no proliferation was observed after 3 days in culture. The addition of R1881, however, helped sustain cell proliferation and increased the calculated doubling time by twofold at the concentration used ( 1 or 5 nM R1881).

To determine whether $\mathrm{E} 8$ cells possessed the ability to induce tumors in vivo, $1 \times 10^{6} \mathrm{E} 8$ cells were subcutaneously injected into male NOD.SCID mice. Tumor incidence was observed to be $100 \%$ (6/6 mice). Histological analysis showed that the grafts indeed resembled adenocarcinoma containing glandular structures composed of multiple layers of epithelial cells expressing AR and CK8 (Fig. 4E) and breaching into the surrounding stroma. Positivity for keratin 5 (CK5), a basal epithelial cell maker, could be seen in cells lining the lumen. Grafts also stained positive for Ki67.

\section{Expression of noggin in CAFs increases anaplastic growth in E8, but not in CE1 cells}

To study how noggin over-expression in CAF cells might influence tumor growth in vivo, we mixed transduced CAF cells with non-transduced epithelial cells, androgendependent E8, and castration-resistant cE1 at a 2:1 ratio and subcutaneously injected into male NOD.SCID mice. Tumor incidence was $100 \%$ in 12 mice. Grafts containing transduced CAF and cE1 cells were generally larger than grafts formed of CAF and E8 cells. While we observed a trend of grafts containing $\mathrm{CAF} /$ noggin cells being larger
A
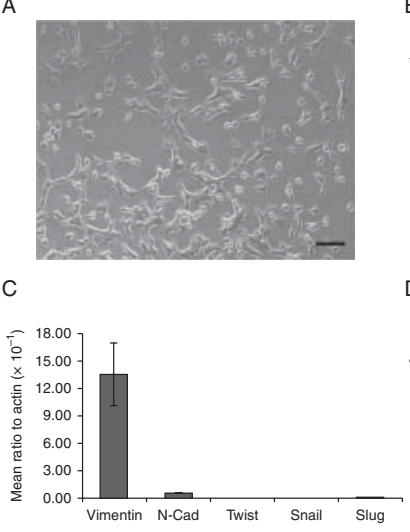

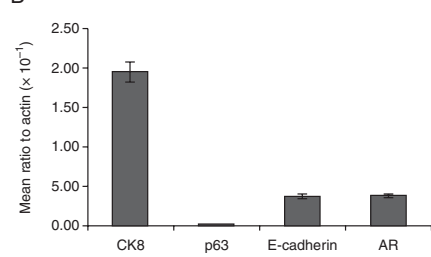

D

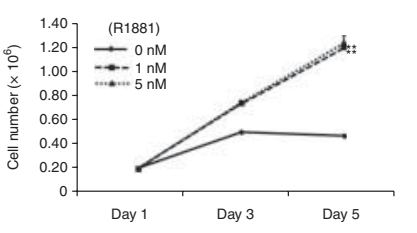

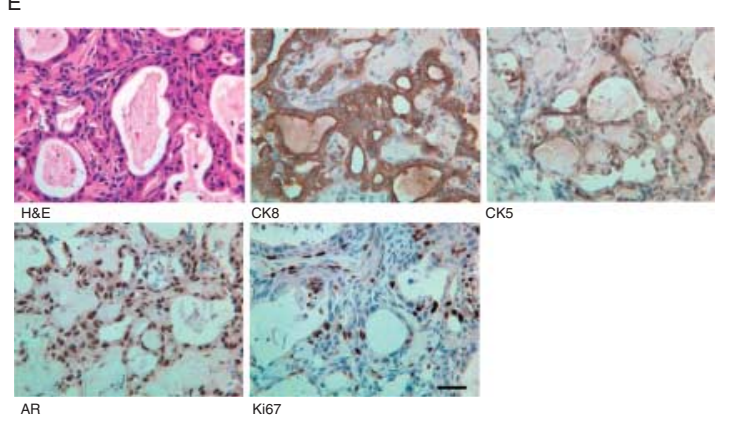

\section{Figure 4}

Characteristics of a new murine prostatic epithelial cell line, E8. (A) $100 \times$ brightfield micrograph of E8 cells in their normal growth media showing polygon-shaped morphology and lack of cell attachment. Bar, $100 \mu \mathrm{m}$. (B) Epithelial (CK8, p63, E-cadherin), and (C) fibroblast (vimentin, $\mathrm{N}$-cadherin) and EMT (Twist, Snail, Slug) expression profile for E8 cell lines as observed by real-time PCR analysis. Shown are mean values \pm S.D.
(D) Comparison of E8 growth rate when cultured in SFM containing 0 , 1, or $5 \mathrm{nM}$ R1881. Cultures containing androgen had significantly higher cell numbers than those that were androgen free. (E) $400 \times$ micrographs of $\mathrm{H} \& \mathrm{E}$ and IHC staining of sections of E8 grafts for AR, CK8, CK5, and Ki67 showing an adenocarcinoma-like morphology. Bar, $50 \mu \mathrm{m}$. ${ }^{*} P<0.01$. http://erc.endocrinology-journals.org DOI: 10.1530/ERC-13-0100
(C) 2013 Society for Endocrinology Printed in Great Britain
Published by Bioscientifica Ltd. 
than those with corresponding tumor cells mixed with $\mathrm{CAF} /$ control, there was only a significant difference in tumor mass in $\mathrm{E} 8+\mathrm{CAF} /$ noggin grafts vs $\mathrm{E} 8+\mathrm{CAF} /$ control (Fig. 5A). Interestingly, both H\&E and CK8 IHC staining showed a striking difference in morphology between E8+ $\mathrm{CAF} /$ noggin and $\mathrm{E} 8+\mathrm{CAF} /$ control grafts. Little to no glandular-like structures formed in $\mathrm{E} 8+\mathrm{CAF} /$ noggin grafts vs a mix of both structured and non-structured growth in $\mathrm{E} 8+\mathrm{CAF} /$ control grafts (Fig. 5B). In grafts containing cE1, only one $\mathrm{CE} 1+\mathrm{CAF} /$ noggin graft lacked any glandular-like structures whereas the other $\mathrm{cE} 1+\mathrm{CAF} /$ noggin grafts as well as all $\mathrm{cE} 1+\mathrm{CAF} /$ control grafts contained both areas of glandular-like structures and areas of non-structured growth (Fig. 5B). In all grafts formed from either E8 or cE1 cells, glandular-like structures stained positive for both CK8 and AR. Vimentin staining was mainly localized to the stroma with weakly staining patches in CK8-positive, AR-positive cells (Fig. 5B). Nuclear AR staining, however, was stronger in grafts containing $\mathrm{CAF} /$ control than in CAF/noggin cells (Fig. 5B).

In areas containing no glandular-like structures, CK8positive cells with vimentin expression and large nuclei were detected. This observation led us to speculate that these cells might constitute anaplastic tumor cells often observed in high-grade tumors (Fig. 5B). To determine whether these cells co-express both CK8 and vimentin, we did co-immunofluorescence staining that showed these areas expressed variable levels of CK8 that co-localized with vimentin (Fig. 6, arrow). Thus, we found that mixing $\mathrm{CAF} /$ noggin cells with $\mathrm{E} 8$ increased the prevalence of de-differentiated cells exhibiting decreased presence of glandular-like structures in the resulting tumors. In cE1 cells, we saw that CAF/noggin cells showed this effect in one graft while the other grafts did not show significant differences in pathology compared with cE1+CAF/ control. Ki67 staining was high in all grafts with at least $20 \%$ of cells staining positive (Fig. $5 \mathrm{~B}$ ) and no significant differences were found among the grafts.

As we did for cE1/control and cE1/noggin grafts, we determined whether there were any alterations to be found in the vascularization of the grafts composed of $\mathrm{CAF} /$ control and CAF/noggin by CD31 immunohistochemistry. Figure $7 \mathrm{~A}$ and $\mathrm{B}$ illustrates the observation that $\mathrm{CD} 31$ staining in $\mathrm{E} 8+\mathrm{CAF} /$ noggin grafts was slightly but not significantly decreased relative to $\mathrm{E} 8+\mathrm{CAF} / \mathrm{con}$ trol grafts. However, in cE1 $+\mathrm{CAF} /$ noggin grafts, the level of positive staining was moderate, but significantly lower than in $\mathrm{cE} 1+\mathrm{CAF} / \mathrm{control}$. This was unexpected as the tumors in the $\mathrm{CE} 1+\mathrm{CAF} /$ noggin group were trending slightly but not significantly larger. We can only surmise that although the vascularization was reduced in cE1+ $\mathrm{CAF} /$ noggin, there was enough angiogenesis to support growth until the endpoint of this experiment. Perhaps if we had prolonged tumor growth, negative effects of reduced angiogenesis would have transpired.

\section{Discussion}

BMPs are increasingly utilized for many therapeutic purposes (Kim \& Choe 2011, Even et al. 2012, Pensak \& Lieberman 2013). While it is important to define specific effects of individual BMPs, there is also a justification to study them collectively because BMPs as a group bind to the same set of receptors and are subject to the same group of antagonists. In order to understand how the overall impact of BMP signaling might affect tumor cells, we took advantage of the ability of noggin to inhibit a number of different BMPs, particularly 2, 4, and 7. Many studies have attempted to interrogate how noggin over-expression in cancer cells may affect tumor progression; however, a majority of these studies focused on the bone microenvironment, using cell lines isolated from metastatic lesions (Feeley et al. 2005, Virk et al. 2010, Secondini et al. 2011). The focus of our current study was to evaluate noggin over-expression in cell lines derived from the prostate tumors at the primary organ site but representing both androgen-dependent and castration-resistant growth phases. Thus, we used two cell lines: androgen-dependent E8 cells and castration-resistant cE1 cells isolated from the prostate of the conditional Pten knockout model of prostate cancer. The E8 cell line is first characterized in this study and we demonstrate that E8 is a cell line that, despite having some mesenchymal-like characteristics perhaps due to its transformed state, expresses epithelial markers and little EMT markers, such as Twist, Snail, and Slug, and thus is not actively undergoing EMT. This phenotype is stable in culture and when subcutaneously injected forms adenocarcinoma in vivo. E8 is also androgen dependent as it has limited proliferative capacity without androgen.

The study was formulated to address two biologically relevant issues: i) the effect of noggin over-expression on castration-resistant $\mathrm{CE} 1$ prostate tumor cells and ii) the effect of noggin over-expression in the fibroblastic stroma on the grafts induced by E8 or cE1 cells. We demonstrate that over-expression of noggin in cE1 cells results in inhibition of in vitro cell proliferation. This is consistent with our previous findings that BMP7 is able to stimulate growth of human prostate cancer cell lines (Yang et al. 2005, 2006). It was demonstrated that BMP7 stimulates

Published by Bioscientifica Ltd 


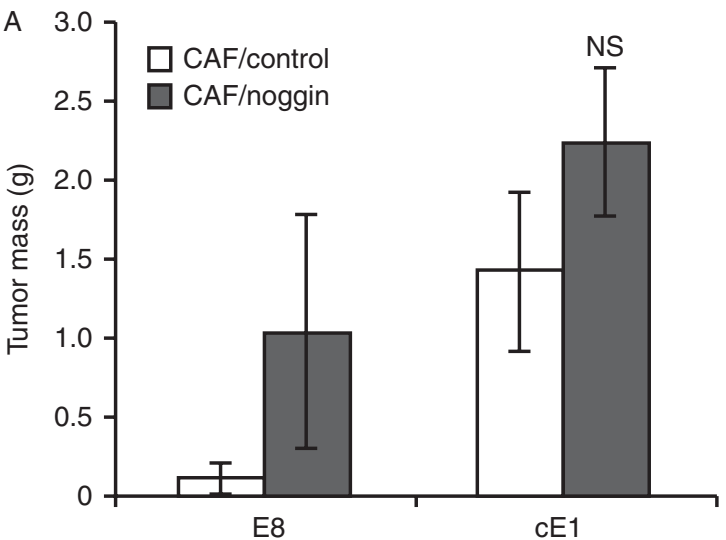

$H \& E$

CK8
E8+CAF/C
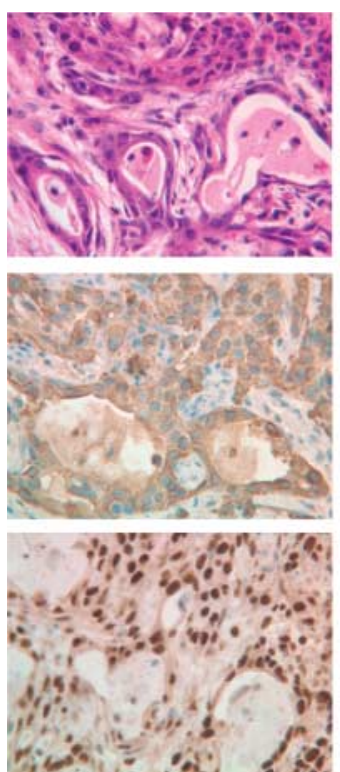

AR
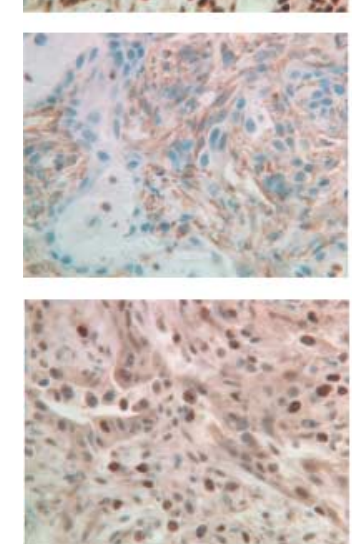

$\mathrm{E} 8+\mathrm{CAF} / \mathrm{N}$
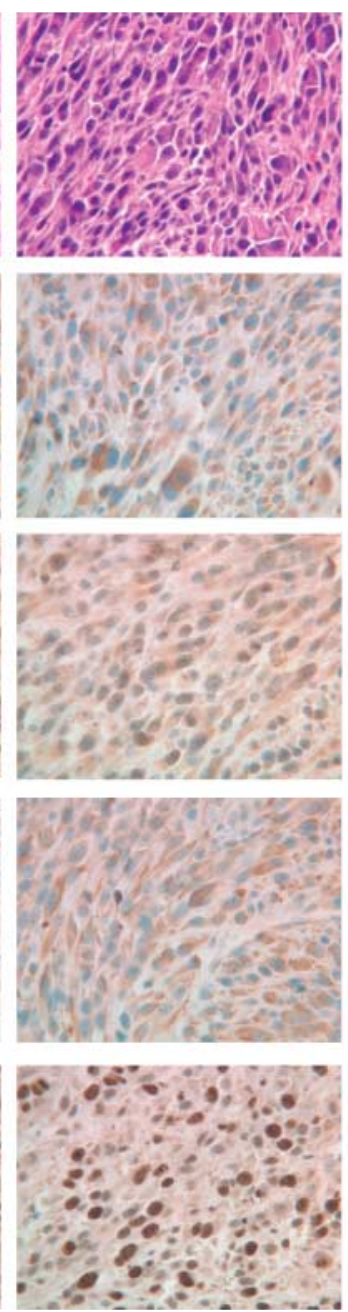

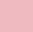

. 

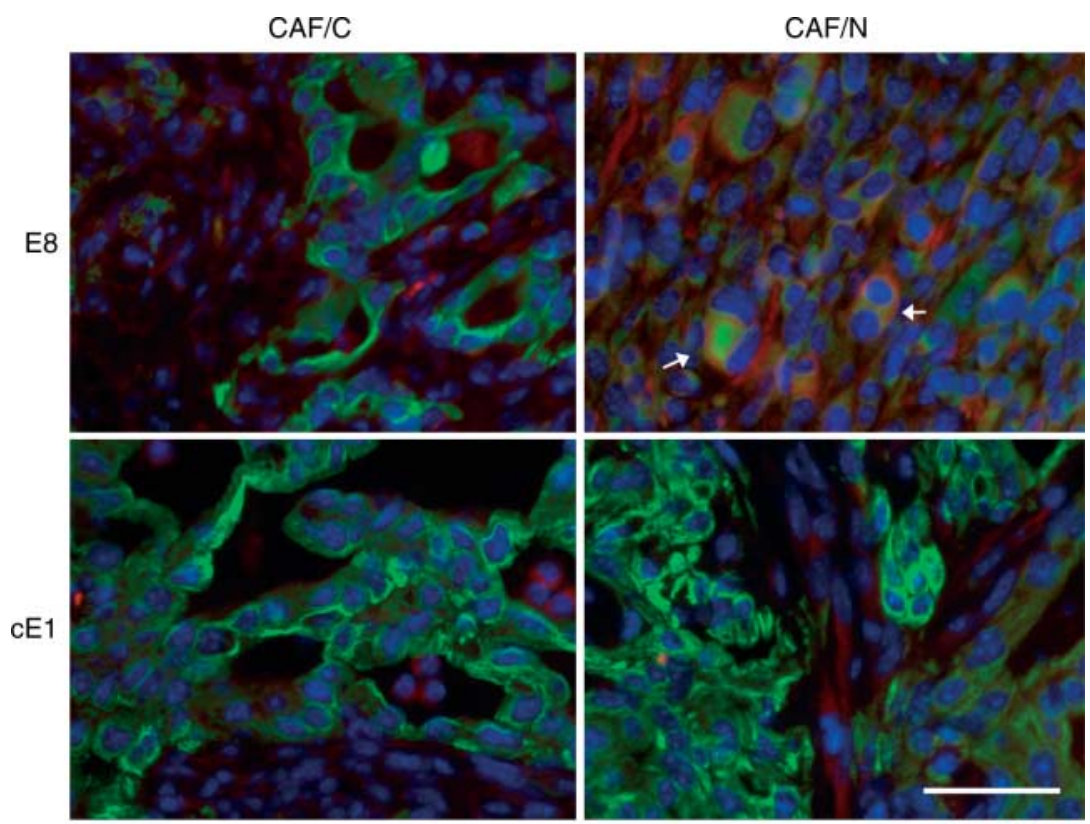

CK8+Vim

\section{Figure 6}

Over-expression of noggin in CAF cells promotes anaplastic growth of tumor cells. Co-immunofluorescence staining showing presence of

proliferation, in part, by suppressing apoptosis via survivin, and it is conceivable that noggin would be able to counter this mechanism. It was, however, interesting to find that noggin was able to increase migration in cE1 epithelial cells, although not that of the fibroblast cells. The propensity of cE1 to migrate faster in the presence of de-differentiated cells with CK8 (green) and vimentin (red) taken at $200 \times$. Arrow, co-localized expression. Bar, $25 \mu \mathrm{m}$.

noggin is not consistent with our findings in BMP7-treated human cell lines, but, as noted earlier, studies of BMPs have had multiple inconsistent results concerning migration of a particular cell line being tested (Buijs et al. 2007, Ye et al. 2007, Benelli et al. 2010). Further work with the cE1 line and CAFs would need to be done to
A

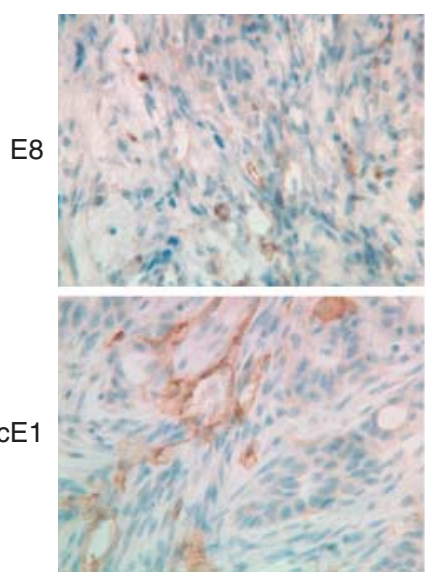

$\mathrm{CAF} / \mathrm{N}$

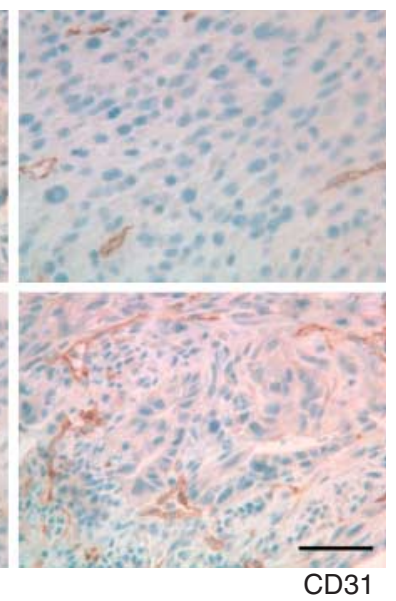

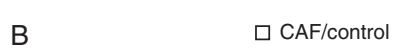

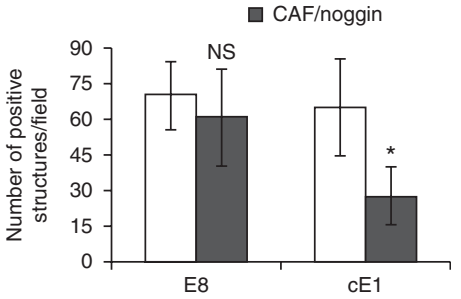

\section{Figure 7}

Forced expression of noggin in CAF cells decreased vascularization in CE1 grafts but not E8 grafts. (A) $400 \times$ micrographs showing CD31 IHC staining of E8 + CAF/control, E8 + CAF/noggin, $\mathrm{CE} 1+\mathrm{CAF} /$ control, and $\mathrm{CE} 1+$ $\mathrm{CAF} /$ noggin grafts. Bar, $50 \mu \mathrm{m}$. (B) Bar graph showing quantitation of CD31
IHC staining. Shown are mean values \pm s.D. The number of positive structure in three independent $400 \times$ fields in each graft was counted and averaged. ${ }^{*} P<0.05$. NS, not significant. http://erc.endocrinology-journals.org DOI: 10.1530/ERC-13-0100
(C) 2013 Society for Endocrinology Printed in Great Britain 
understand how and why noggin promotes migration in cE1 and not CAF.

The implication that inhibition of BMP functions decreases proliferation is mitigated by our in vivo findings that cE1/noggin grafts are significantly larger and more proliferative than the corresponding controls. The disparity between the in vitro and in vivo results perhaps highlight the importance of the tumor microenvironment in modulating the effects of noggin. Although no obvious differences were apparent in the morphology of the tumors formed in the presence or absence of the BMP inhibitor, it seems logical to conclude that, in vivo, the overall effect of BMP signaling on cE1 is anti-proliferative. A tumor-suppressive effect of BMP on cE1 cells is consistent with many studies that demonstrate in human prostate cancer, expression of BMPs, or its receptors is reduced or lost (Kim et al. 2000, Thomas et al. 2001, Masuda et al. 2004) during tumor progression at the primary site. Also, the idea of BMP as a tumor suppressor is consistent with a recent study showing that prostate epithelial specific knock out of SMAD signaling in the conditional Pten prostate cancer model increases aggressiveness of tumor progression and occurrence of metastasis; BMP/transforming growth factor $\beta$ signaling may actually act as a barrier to metastasis in the mouse model (Ding et al. 2011).

As we have evidence that BMP is able to stimulate tumor-promoting properties of CAF cells in vitro (Yang et al. 2008), we also over-expressed noggin in CAF cells of the tumor model to determine their effect on tumor growth induced by either E8 or cE1 cells. We presumed that reconstituting a tumor with these cellular components might recapitulate tumor behavior, at least in part, to that encountered in vivo. The most noticeable difference we detected is the anaplastic morphology seen in $100 \%(3 / 3)$ of $\mathrm{E} 8$ grafts with CAF/noggin indicative of a higher grade tumor than that seen in the case of $\mathrm{E} 8+$ $\mathrm{CAF} /$ controls. $\mathrm{E} 8+\mathrm{CAF} /$ noggin grafts also displayed greater tumor mass compared with $\mathrm{CAF} /$ control counterparts. These results support an anti-oncological role for BMPs consistent with the data seen in cE1/noggin grafts. Notably, the castration-resistant tumor cell line, cE1, only had one graft with $\mathrm{CAF} /$ noggin that had a similar morphology to that observed with the $\mathrm{E} 8+\mathrm{CAF} /$ noggin group. cE1 + CAF/noggin, unlike E8 + CAF/noggin, did not show a remarkable increase in tumor growth when compared with its $\mathrm{CAF} /$ controls, although cE1 + CAF grafts did grow larger than cE1 only grafts, as expected (Orimo et al. 2005).
There are a number of possible reasons for the contrasting results we see with E8 and cE1 cells. Perhaps the ability of CAF/noggin to induce anaplasia in E8 cells is facilitated by the partially de-differentiated state that existed originally. Another possibility is that the CAFs used, which are from an androgen-dependent tumor, were not be able to promote tumorigenesis in cE1 cells as well as in E8 cells because cE1 cells were isolated from a castration-resistant tumor and thus a different microenvironment may be nonpermissive. Further studies are needed to determine whether CAFs isolated from a castration-resistant tumor are differentially influencing cE1 cells. Another point is that Morrissey et al. (2010) recently noted that their results with BMP7 were dependent on the androgen dependent/castration-resistant status of the human LNCaP and C4-2B cell lines. Thus, it is possible that the effect of noggin may also be dependent on the differing levels of androgen sensitivities of E8 and cE1 cells. Nonetheless, it is clear that inhibition of BMP signaling in CAF cells can increase their ability to support prostate tumor progression. In other words, the results obtained may imply that BMP has a generally negative impact on growth or re-growth of prostate tumor at a primary site but the cellular parameters or the mechanisms that drive this important function remain to be elucidated. However, our findings on the striking differences in the E8 and cE1 grafts mixed with $\mathrm{CAF} /$ noggin also underscore that the overall effect of BMPs may not be scrutinized in a biologically meaningful manner by solely focusing on the tumor cells without considering the context of the stromal components. The overall effect of BMP signaling on prostate tumors is likely to be an amalgam of its effect on the tumor cells, on the stromal cells in the tumor microenvironment, and on the heterotypic interactions that occur between the various cell types present in the tumor organ.

\section{Declaration of interest}

The authors declare that there is no conflict of interest that could be perceived as prejudicing the impartiality of the research reported.

\section{Funding}

This study was funded by NIH R01 CA59705 and NIH R01 CA113392 (P Roy-Burman) and in part by NIH training grant T32 Al07078 (L K Pham).

\section{Acknowledgements}

The authors would like to acknowledge Emanuelle Faure-Kumar of the UCLA Vector Core (supported by JCCC/P30 CA016042 and CURE/P30

Published by Bioscientifica Ltd 
DK041301), Michelle MacVeigh of the Cell and Tissue Imaging Core of the USC Research Center for Liver Diseases (supported by NIH grants P30 DK048522 and S10 RR022508), Lora Barsky of the USC Flow Cytometry Core Facility (supported in part by NCI CCSG award number P30CA014089), and all members of the Roy-Burman Laboratory for their assistance.

\section{References}

Avsian-Kretchmer O \& Hsueh AJ 2004 Comparative genomic analysis of the eight-membered ring cystine knot-containing bone morphogenetic protein antagonists. Molecular Endocrinology 18 1-12. (doi:10.1210/me. 2003-0227)

Benelli R, Monteghirfo S, Vene R, Tosetti F \& Ferrari N 2010 The chemopreventive retinoid 4HPR impairs prostate cancer cell migration and invasion by interfering with FAK/AKT/GSK3 $\beta$ pathway and $\beta$-catenin stability. Molecular Cancer 9 142. (doi:10.1186/1476-45989-142)

Buijs JT, Rentsch CA, van der Horst G, van Overveld PG, Wetterwald A, Schwaninger R, Henriquez NV, Ten Dijke P, Borovecki F, Markwalder R et al. $2007 \mathrm{BMP} 7$, a putative regulator of epithelial homeostasis in the human prostate, is a potent inhibitor of prostate cancer bone metastasis in vivo. American Journal of Pathology 171 1047-1057. (doi:10.2353/ ajpath.2007.070168)

Dai J, Kitagawa Y, Zhang J, Yao Z, Mizokami A, Cheng S, Nor J, McCauley LK, Taichman RS \& Keller ET 2004 Vascular endothelial growth factor contributes to the prostate cancer-induced osteoblast differentiation mediated by bone morphogenetic protein. Cancer Research 64 994-999. (doi:10.1158/0008-5472.CAN-03-1382)

Dai J, Keller J, Zhang J, Lu Y, Yao Z \& Keller ET 2005 Bone morphogenetic protein- 6 promotes osteoblastic prostate cancer bone metastases through a dual mechanism. Cancer Research 65 8274-8285. (doi:10.1158/0008-5472.CAN-05-1891)

Ding Z, Wu CJ, Chu GC, Xiao Y, Ho D, Zhang J, Perry SR, Labrot ES, Wu X, Lis $\mathrm{R}$ et al. 2011 SMAD4-dependent barrier constrains prostate cancer growth and metastatic progression. Nature 470 269-273. (doi:10.1038/ nature09677)

Dull T, Zufferey R, Kelly M, Mandel RJ, Nguyen M, Trono D \& Naldini L 1998 A third generation lentivirus vector with a conditional packaging system. Journal of Virology $\mathbf{7 2}$ 8463-8471.

Even J, Eskander M \& Kang J 2012 Bone morphogenetic protein in spine surgery: current and future uses. Journal of the American Academy of Orthopaedic Surgeons 20 547-552. (doi:10.5435/JAAOS-20-09-547)

Feeley BT, Gamradt SC, Hsu WK, Liu N, Krenek L, Robbins P, Huard J \& Lieberman JR 2005 Influence of BMPs on the formation of osteoblastic lesions in metastatic prostate cancer. Journal of Bone and Mineral Research 20 2189-2199. (doi:10.1359/JBMR.050802)

Feeley BT, Krenek L, Liu N, Hsu WK, Gamradt SC, Schwarz EM, Huard J \& Lieberman JR 2006 Overexpression of noggin inhibits BMP-mediated growth of osteolytic prostate cancer lesions. Bone 38 154-166. (doi:10.1016/j.bone.2005.07.015)

Groppe J, Greenwald J, Wiater E, Rodriguez-Leon J, Economides AN, Kwiatkowski W, Affolter M, Vale WW, Izpisua Belmonte JC \& Choe S 2002 Structural basis of BMP signalling inhibition by the cystine knot protein noggin. Nature 420 636-642. (doi:10.1038/nature01245)

Haudenschild DR, Palmer SM, Moseley TA, You Z \& Reddi AH 2004 Bone morphogenetic protein (BMP)-6 signaling and BMP antagonist noggin in prostate cancer. Cancer Research 64 8276-8284. (doi:10.1158/00085472.CAN-04-2251)

Kim M \& Choe S 2011 BMPs and their clinical potentials. BMB Reports 44 619-634. (doi:10.5483/BMBRep.2011.44.10.619)

Kim IY, Lee DH, Ahn HJ, Tokunaga H, Song W, Devereaux LM, Jin D, Sampath TK \& Morton RA 2000 Expression of bone morphogenetic protein receptors type-IA, -IB and -II correlates with tumor grade in human prostate cancer tissues. Cancer Research 60 2840-2844.
Krause C, Guzman A \& Knaus P 2011 noggin. International Journal of Biochemistry \& Cell Biology 43 478-481. (doi:10.1016/j.biocel.2011. 01.007)

Kwon H, Kim HJ, Rice WL, Subramanian B, Park SH, Georgakoudi I \& Kaplan DL 2010 Development of an in vitro model to study the impact of BMP-2 on metastasis to bone. Journal of Tissue Engineering and Regenerative Medicine 4 590-599. (doi:10.1002/term.268)

Liao CP, Liang M, Cohen MB, Flesken-Nikitin A, Jeong JH, Nikitin AY \& Roy-Burman P 2010 Mouse prostate cancer cell lines established from primary and postcastration recurrent tumors. Hormones \& Cancer 1 44-54. (doi:10.1007/s12672-009-0005-y)

Lim M, Zhong C, Yang S, Bell AM, Cohen MB \& Roy-Burman P 2009 Runx2 regulates survivin expression in prostate cancer cells. Laboratory Investigation 90 222-233. (doi:10.1038/labinvest.2009.128)

Masuda H, Fukabori Y, Nakano K, Shimizu N \& Yamanaka H 2004 Expression of bone morphogenetic protein-7 (BMP-7) in human prostate. Prostate 59 101-106. (doi:10.1002/pros.20030)

Morrissey C, Brown LG, Pitts TE, Vessella RL \& Corey E 2010 Bone morphogenetic protein 7 is expressed in prostate cancer metastases and its effects on prostate tumor cells depend on cell phenotype and the tumor microenvironment. Neoplasia 12 192-205.

Orimo A, Gupta PB, Sgroi DC, Arenzana-Seisdedos F, Delaunay T, Naeem R, Carey VJ, Richardson AL \& Weinberg RA 2005 Stromal fibroblasts present in invasive human breast carcinomas promote tumor growth and angiogenesis through elevated SDF-1/CXCL12 secretion. Cell 121 335-348. (doi:10.1016/j.cell.2005.02.034)

Pensak MJ \& Lieberman JR 2013 Gene therapy for bone regeneration. Current Pharmaceutical Design 19 3466-3473. (doi:10.2174/ 1381612811319190012)

Reinhold MI, Abe M, Kapadia RM, Liao Z \& Naski MC 2004 FGF18 represses noggin expression and is induced by calcineurin. Journal of Biological Chemistry 279 38209-38219. (doi:10.1074/jbc.M404855200)

Schwaninger R, Rentsch CA, Wetterwald A, van der Horst G, van Bezooijen RL, van der Pluijm G, Lowik CW, Ackermann K, Pyerin W, Hamdy FC et al. 2007 Lack of noggin expression by cancer cells is a determinant of the osteoblast response in bone metastases. American Journal of Pathology $\mathbf{1 7 0}$ 160-175. (doi:10.2353/ajpath.2007.051276)

Secondini C, Wetterwald A, Schwaninger R, Thalmann GN \& Cecchini MG 2011 The role of the BMP signaling antagonist noggin in the development of prostate cancer osteolytic bone metastasis. PLOS ONE 6 e16078. (doi:10.1371/journal.pone.0016078)

Shaw A, Gipp J \& Bushman W 2009 Exploration of Shh and BMP paracrine signaling in a prostate cancer xenograft. Differentiation 79 41-47. (doi:10.1016/j.diff.2009.08.009)

Song K, Krause C, Shi S, Patterson M, Suto R, Grgurevic L, Vukicevic S, van Dinther M, Falb D, Ten Dijke P et al. 2010 Identification of a key residue mediating bone morphogenetic protein (BMP)- 6 resistance to noggin inhibition allows for engineered BMPs with superior agonist activity. Journal of Biological Chemistry 285 12169-12180. (doi:10.1074/ jbc.M109.087197)

Thomas R, True LD, Lange PH \& Vessella RL 2001 Placental bone morphogenetic protein (PLAB) gene expression in normal, pre-malignant and malignant human prostate: relation to tumor development and progression. International Journal of Cancer 93 47-52. (doi:10.1002/ijc.1291)

Virk MS, Petrigliano FA, Liu NQ, Chatziioannou AF, Stout D, Kang CO, Dougall WC \& Lieberman JR 2009 Influence of simultaneous targeting of the bone morphogenetic protein pathway and RANK/RANKL axis in osteolytic prostate cancer lesion in bone. Bone 44 160-167. (doi:10.1016/j.bone.2008.09.009)

Virk MS, Alaee F, Petrigliano FA, Sugiyama O, Chatziioannou AF, Stout D, Dougall WC \& Lieberman JR 2010 Combined inhibition of the BMP pathway and the RANK-RANKL axis in a mixed lytic/blastic prostate cancer lesion. Bone 48 578-587. (doi:10.1016/j.bone.2010.11.003)

Winkler DG, Yu C, Geoghegan JC, Ojala EW, Skonier JE, Shpektor D, Sutherland MK \& Latham JA 2004 noggin and sclerostin bone http://erc.endocrinology-journals.org

DOI: 10.1530/ERC-13-0100
(C) 2013 Society for Endocrinology Printed in Great Britain 
morphogenetic protein antagonists form a mutually inhibitory complex. Journal of Biological Chemistry 279 36293-36298. (doi:10.1074/jbc.M400521200)

Yanagita M 2005 BMP antagonists: their roles in development and involvement in pathophysiology. Cytokine \& Growth Factor Reviews 16 309-317. (doi:10.1016/j.cytogfr.2005.02.007)

Yang S, Zhong C, Frenkel B, Reddi AH \& Roy-Burman P 2005 Diverse biological effect and Smad signaling of bone morphogenetic protein 7 in prostate tumor cells. Cancer Research 65 5769-5777. (doi:10.1158/ 0008-5472.CAN-05-0289)

Yang S, Lim M, Pham LK, Kendall SE, Reddi AH, Altieri DC \& Roy-Burman P 2006 Bone morphogenetic protein 7 protects prostate cancer cells from stress-induced apoptosis via both Smad and c-Jun $\mathrm{NH}_{2}$-terminal kinase pathways. Cancer Research 66 4285-4290. (doi:10.1158/0008-5472. CAN-05-4456)
Yang S, Pham LK, Liao CP, Frenkel B, Reddi AH \& Roy-Burman P 2008 A novel bone morphogenetic protein signaling in heterotypic cell interactions in prostate cancer. Cancer Research 68 198-205. (doi:10.1158/0008-5472.CAN-07-5074)

Ye L, Lewis-Russell JM, Kynaston H \& Jiang WG 2007 Endogenous bone morphogenetic protein-7 controls the motility of prostate cancer cells through regulation of bone morphogenetic protein antagonists. Journal of Urology 178 1086-1091. (doi:10.1016/j.juro. 2007.05.003)

Yuen HF, Chan YP, Cheung WL, Wong YC, Wang X \& Chan KW 2008 The prognostic significance of BMP-6 signaling in prostate cancer. Modern Pathology 21 1436-1443. (doi:10.1038/modpathol.2008.94)

Zufferey R, Dull T, Mandel RJ, Bukovsky A, Quiroz D, Naldini L \& Trono D 1998 Self inactivating lentivirus factor for safe and efficient in vivo gene delivery. Journal of Virology $\mathbf{7 2}$ 9873-9880.

Received in final form 23 August 2013

Accepted 16 September 2013

Made available online as an Accepted Preprint

16 September 2013
(C) 2013 Society for Endocrinology Printed in Great Britain
Published by Bioscientifica Ltd. 